\title{
Subependymomas of the lateral ventricle: Tumor recurrence correlated with increased Ki-67 labeling index
}

M aria Koutourousiou, N. Georgakoulias, G. Kontogeorgos ${ }^{1}$ A. Seretis

Departments of Neurosurgery and ${ }^{1}$ Pathology, Athens General Hospital, Athens, Greece

Address for correspondence:

Dr. Maria Koutourousiou,

Department of Neurosurgery,

$4^{\text {th }}$ Floor KO FKA Building,

G. Gennimatas, Athens General

Hospital, 154 Messogion Ave,

GR-115 27 Athens, Greece.

E-mail: mkoutourousiou@ yahoo.gr

\begin{abstract}
Abstrad
Subependymomas of the lateral ventricles are rare tumors. We present two patients with subependymomas of the lateral ventricle, who underwent gross total resection of the tumor via transcallosal approach. The patient, with increased Ki-67 labeling index had recurrence of tumor two years after the initial operation. We emphasize at the risk of recurrence which is probably correlated with Ki-67 labeling index.
\end{abstract}

Key words: Intraventricular tumors, magnetic resonance imaging, subependymomas

DOI: $10.4103 / 0028-3886.51293$

\section{Introduction}

Subependymomas are rare, noninvasive, slow-growing tumors. Most frequently, they are located in the fourth $(50-60 \%)$ and lateral ventricles (30-40\%). ${ }^{[1]}$ Rarely they arise in the third ventricle, septum pelludicum and the spinal cord. ${ }^{[2-4]}$ Histologically, subependymomas correspond, according to World Health Organization (WHO) classification, to Grade I tumors ${ }^{[5]}$ An increasing number of asymptomatic subependymomas has been detected with the introduction of computed tomography (CT) and magnetic resonance imaging (MRI). Due to their rarity and variable imaging characteristics, reliable preoperative diagnosis remains challenging.

In this paper we present two patients with subependymoma of the lateral ventricle and emphasize the risk of recurrence may be correlated with Ki-67 labeling index.

\section{Case Report}

\section{Case 1}

A 32-year-old woman presented with altered level of consciousness, nausea, vomiting and bilateral papilledema in 2003. MRI revealed a right lateral intraventricular mass with obstructive hydrocephalus. The lesion was of low signal intensity on the T1-weighted images and showed no enhancement after Gadolinium (Gd) administration [Figure 1a]. Patient had initial ventriculoperitoneal shunt followed by gross total resection of the lesion via a transcallosal approach [Figure 1b]. Postoperatively the patient became asymptomatic. Histopathology revealed a subependymoma with hypocellular areas containing a dense pink fibrillary matrix with microcyst formation. Ki-67 (clone MIB-1) immunostaining revealed labeling indices approximately 3\%, reaching $10 \%$ at the most hypercellular areas, suggesting a high proliferative lesion [Figures 2a and 2b]. Mitoses were not remarkable. The patient was followed up with regular MRIs. In August 2005 she presented with a recurrent lesion, measuring $2 \mathrm{~cm}$ in diameter on MRI [Figure 3]. The size of the mass remained unchanged during the follow-up course. As she is asymptomatic we decided not to operate and her with regular MRIs.

\section{Case 2}

A 25-year-old woman presented with headache, gait disturbance (ataxia) and bilateral papilledema in August 2004. MRI revealed a right lateral intraventricular mass which was isointense on the T1-weighted images and hyperintense on T2-weighted images and there was no contrast enhancement after $\mathrm{Gd}$ administration [Figure 4a]. The patient underwent a gross total resection of the mass via a transcallosal approach [Figure 4b]. Postoperatively 


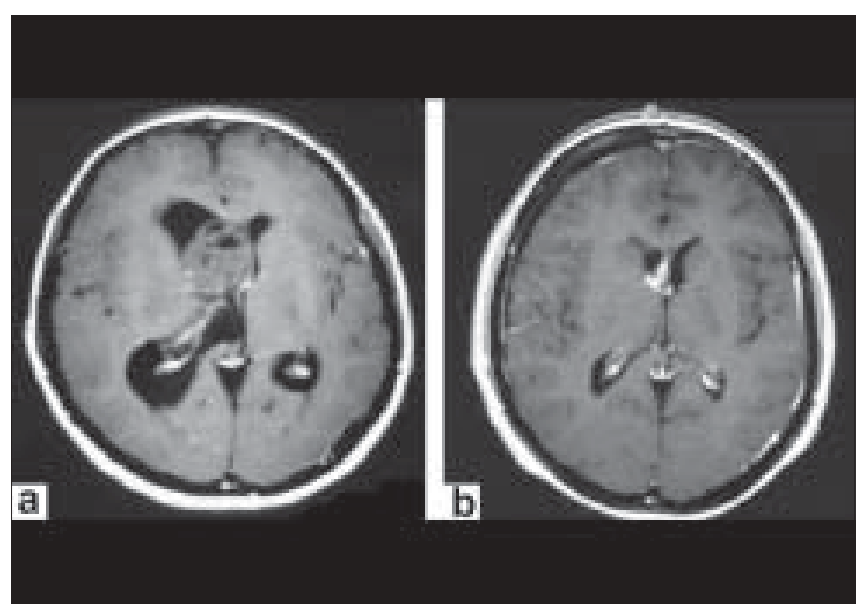

Figure 1: Axial T1-weighted images of our first patient after administration of Gd, preoperatively, with no contrast enhancement (a) and postoperatively, after gross total resection of the lesion via transcallosal approach, with no sign of residual mass (b)

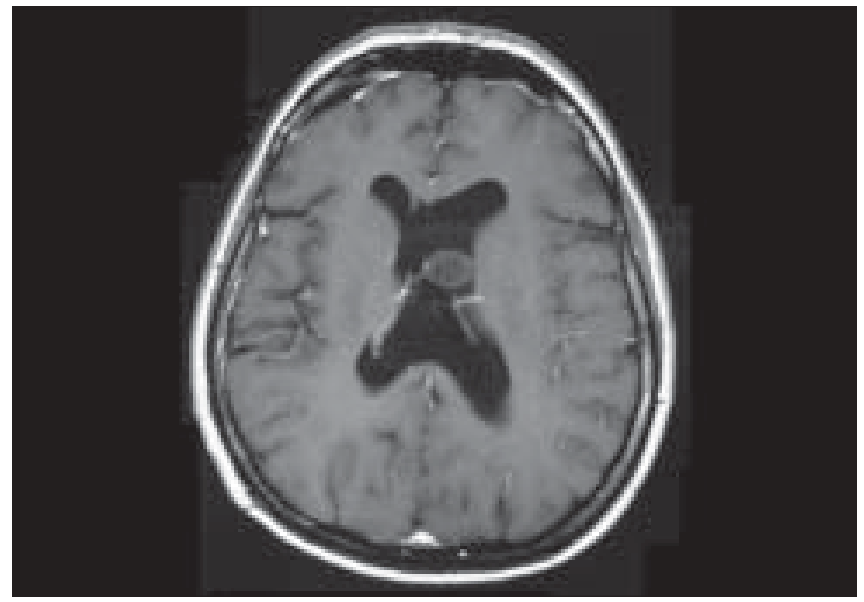

Figure 3: Axial contrast T1-weighted image of the first patient, two years after operation, demonstrates the recurrent tumor

the patient became asymptomatic. The histological examination showed typical features of subependymoma. The Ki-67 labeling index was less than $2 \%$. Mitoses were very rare. The patient is asymptomatic with no signs of recurrence at two years of follow-up postoperatively.

\section{Discussion}

Subependymoma is a rare, usually asymptomatic benign, noninvasive, slow-growing neoplasm, with an incidence of $0.2-0.7 \%$ of intracranial tumors. ${ }^{[3,6]}$ Symptoms have been shown to directly correlate with tumor size and the majority of the symptomatic subependymomas measure $3-5 \mathrm{~cm}$ or greater in size. Patients with symptomatic subependymomas are usually men between the fourth and sixth decades of life and present with signs and symptoms related to cerebrospinal fluid (CSF) obstruction, leading to increased intracranial pressure. The most common symptoms include headache, gait

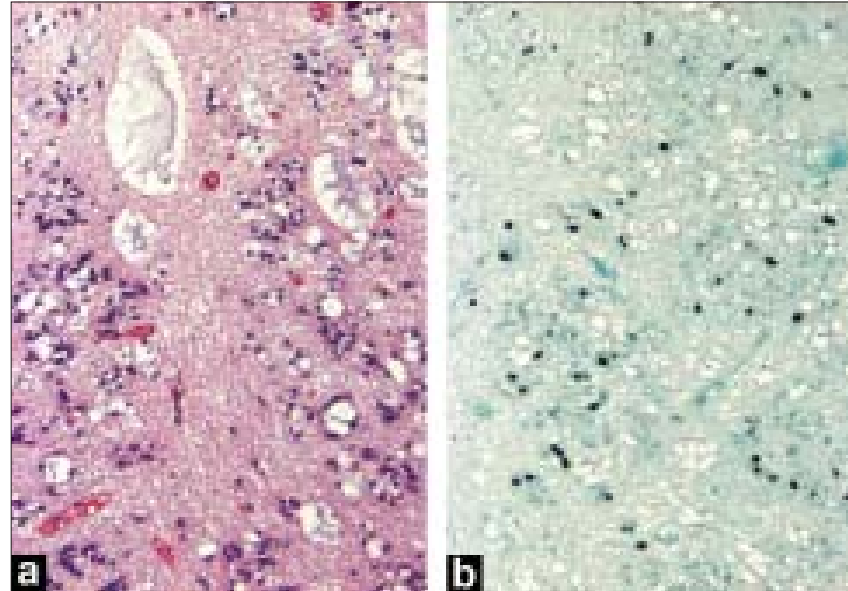

Figure 2: Typical histology of subependymoma composed of cell clusters with small uniform nuclei embedded in a fibrillary matrix showing microcystic degeneration (a: H\&E, $\times 10)$. Increased Ki-67 labeling index reaching $10 \%$ of tumor cells (b: Abidin-biotin-peroxidase complex immunohistochemical technique $\times 10$ )

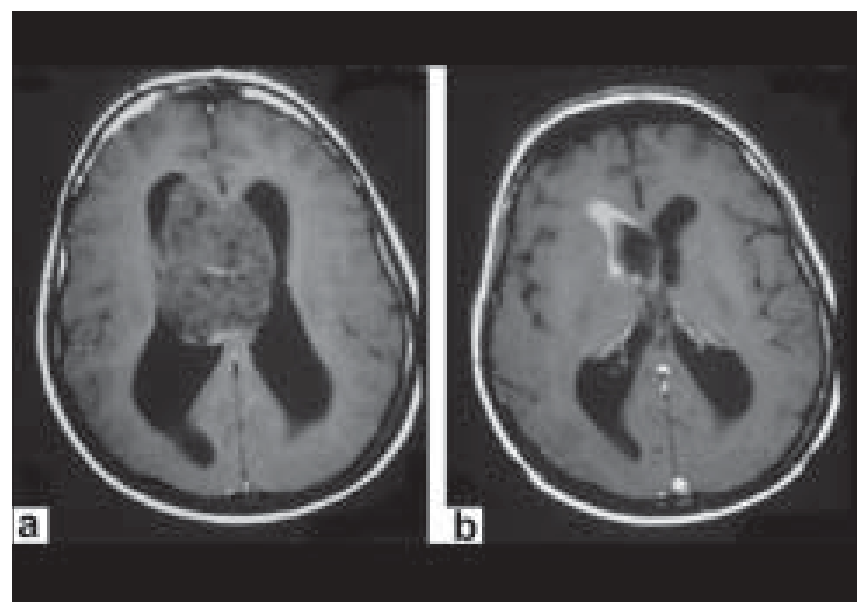

Figure 4: Axial T1-weighted images of the second patient after administration of Gd, preoperatively, revealed an intraventricular mass which was isointense with brain parenchyma with no contrast enhancement (a) and after total excision of the mass (b)

ataxia, vertigo or dizziness, nausea and vomiting. ${ }^{[1,6]}$

On CT most subependymomas appear as a lobular, well-defined intraventricular mass that is hypodense to isodense to brain parenchyma with no periventricular extension. ${ }^{[1-3]}$ Hyperdense subependymomas have rarely been reported. The majority of subependymomas are solid or solid with cystic components and contrast CT usually shows little or no enhancement. ${ }^{[1,6]}$ MRI characteristics of subependymomas include a welldefined solid or mixed solid and cystic intraventricular mass, and the solid component of which is hypo or isontense on T1-weighted images and hyperintense on T2-weighted images. ${ }^{[2,3]}$ Contrast MRIs show little or no enhancement. ${ }^{[1,6,7]}$ However, several cases of subependymomas with enhancement of Gd have been reported. ${ }^{[7]}$ Proton MR Spectroscopy (H-MRS) is 
important for estimating the changes in metabolites, and in the case of subependymoma includes increased choline (Cho) peak, presence of lactate/lipids peaks and decreased N-acetyl aspartate (NAA) and creatine (Cre) peaks. ${ }^{[8]}$ Cho reflects the membrane synthesis and degeneration. Therefore, higher Cho levels occur in more aggressive tumor types. Thallium-201 single photon emission computed tomography (T1-SPECT) has been used to determine the biological activity of the tumor. T1 uptake is related to changes in blood flow. High uptake of T1 without washout in the delayed image is suggestive of malignant nature of the tumors. ${ }^{[9]}$ Subependymomas show no uptake in both early and delayed images. It is clear that the neuroimagining characteristics of subependymoma are non-specific and cannot be used to establish the correct preoperative diagnosis. The differential diagnosis of the lateral ventricle tumors include choroidal plexus papilloma, central neurocytoma, subependymal giant cell astrocytoma, meningioma, metastasis, and ependymoma.

According to WHO subependymoma is classified as a Grade I neoplasm. It is a nodular tumor composed of remnants of ependymal cells in a dense glial fibrillary matrix. Hemosiderin deposits are commonly seen. Microcysts and calcification are frequently observed..$^{[1,4,5]}$ However, the histological origin of subependymoma has remained controversial. A recent report has suggested that the bland microscopic appearance and low Ki-67 (clone MIB-1) labeling index of subependymoma support the hypothesis that it may actually represent a hamartoma rather than a neoplasm. ${ }^{[4]}$ According to Prayson et al., ${ }^{[4]}$ subependymomas with clinical symptoms had slightly higher Ki-67 indices than tumors discovered at autopsy, suggesting that even a slight increase in proliferative rate may affect clinical behavior. According to the WHO Classification of Tumors, ${ }^{[5]}$ subependymomas usually have Ki-67 indices less than 1\%, a fact related to the benign, slow-growing nature of these lesions. In one of our cases, the patient with Ki-67 index of $10 \%$, presented with recurrence thus confirming the value of Ki-67 labeling index as a predictive-proliferative marker, which may indicate aggressive clinical course.

Complete surgical excision is the optimal therapy for symptomatic subependymomas. Interhemispheric transcallosal approach is the preferred one because of the easy access to both lateral ventricles and the absence of cortical incision, which reduce the incidence of postoperative seizures. ${ }^{[3,6,10]}$ The prognosis after total resection of subependymoma is very good, so that postoperative radiation therapy is not recommended. ${ }^{[2]}$ Radiation therapy is advocated in the management of subtotally resected or recurrent masses, at doses of 5,000 cGy or greater. ${ }^{[3]}$ However, the role of radiotherapy is unclear because of the limited number of cases and the short-term follow-up. ${ }^{[4,6]}$

In conclusion, subependymomas of the lateral ventricle are rare, benign tumors and when symptomatic the recommended therapy is total surgical resection via a transcallosal approach. The presence of increased Ki-67 labeling index may indicate aggressive clinical course, suggesting that tumor recurrence is correlated with high Ki-67 index.

\section{References}

1. Nishio S, Morioka T, Mihara F, Fukui M. Subependymoma of the lateral ventricles. Neurosurg Rev 2000;23:98-103.

2. Im SH, Paek SH, Choi YL, Chi JG, Kim DG, Jung HW, et al. Clinicopathological study of seven cases of symptomatic supratentorial subependymoma. J Neurooncol 2003;61:57-67.

3. Lombardi D, Scheithauer BW, Meyer FB, Forbes GS, Shaw EG, Gibney DJ, et al. Symptomatic subependymoma: A clinicopathological and flow cytometric study. J Neurosurg 1991;75:583-8.

4. Prayson F, Suh JH. Subependymomas: Clinicopathologic study of 14 tumors, including comparative MIB-1 immunohistochemical analysis with other ependymal neoplasms. Arch Pathol Lab Med $1999 ; 123: 306-9$

5. Louis DN, Ohgaki H, Wiestler OD, Cavenee WK, editors. World Health Organization Classification of Tumours. Pathology and genetics of tumours of the nervous system. Lyon: IARC; 2007.

6. Matsumara A, Ahyai A, Hori A, Schaake T. Inrtacerebral subependymoma: Clinical and neuropathological analysis with special reference to the possible existence of a less benign variant. Acta Neurochir 1989;96: $15-25$.

7. Silverstein JE, Lenchik L, Stanciu MG, Shimkin PM. MRI on intracranial subependymoma. J Comput Assist Tomogr 1995;19:264-7.

8. Shimizu H, Kumambe T, Hara K, Fujiwara S, Yoshimoto T. Cho level measured by proton magnetic resonance spectroscopy correlate with Ki-67 labeling index in gliomas. Am J Neuroradiol 2000;21:659-65.

9. Ueda T, Kaji Y, Wakisaka S, Hoshi H, Jinnouchi S, Futami S. Time sequential single proton emission computed tomography studies in brain tumors using thallium-201. Eur J Nucl Med 1993;20:138-45.

10. Anderson R, Ghatan S, Feldstein N. Surgical approaches to tumors of the lateral ventricle. Neurosurg Clin N Am 2003;14:509-25.

Accepted on 06-03-2009

Source of Support: Nil, Conflict of Interest: None declared. 